\title{
Photometric observations of RCB stars In the LMC
}

\author{
W.A. Lawson and P.L. Cottrell \\ Mount John University Observatory, \\ Department of Physics, \\ University of Canterbury, \\ Christchurch, New Zealand.
}

We present UBV photometric observations of two R Coronae Borealis (RCB) stars, W Mensae and HV12842, which are members of the Large Magallenic Cloud (LMC). These data have been obtained over the last two years using single-channel photometers on both the $0.6 \mathrm{~m}$ and $1 \mathrm{~m}$ telescopes at Mount John. They form part of an ongoing long-term program to investigate photometric and spectroscopic variations in the hydrogen-deficient carbon (HdC) stars both in the LMC and in our Galaxy.

These two stars show quite different properties, indicative of the nature of these objects. HV12842 (Fig.1) shows semi-regular pulsations not dissimilar to the bright southern RCB star, RY Sgr (see Lawson, Cottrell and Bateson 1988), whereas W Mensae has a V magnitude which seems to have no obvious periodic variations, similar to the variations in $\mathrm{R}$ Coronae Borealis itself. In addition, the $\mathrm{V}$ magnitude curve of HV12842 appears to show beating due to multiple mode pulsations, as the amplitude of the pulsations has changed from $\approx 0.5 \mathrm{mag}$. to $<0.05 \mathrm{mag}$. over a period of about 450 days. Further confirmation of this effect is shown by the recent observations (JD 2447300 onwards) which have a $\mathrm{V}$ amplitude of $\approx 0.1 \mathrm{mag}$.

Lawson,W.A.,Cottrell,P.L.\&Bateson,F.M.1988,Publ.Var.StarSect.R.A.S.N.Z.,14, 38.

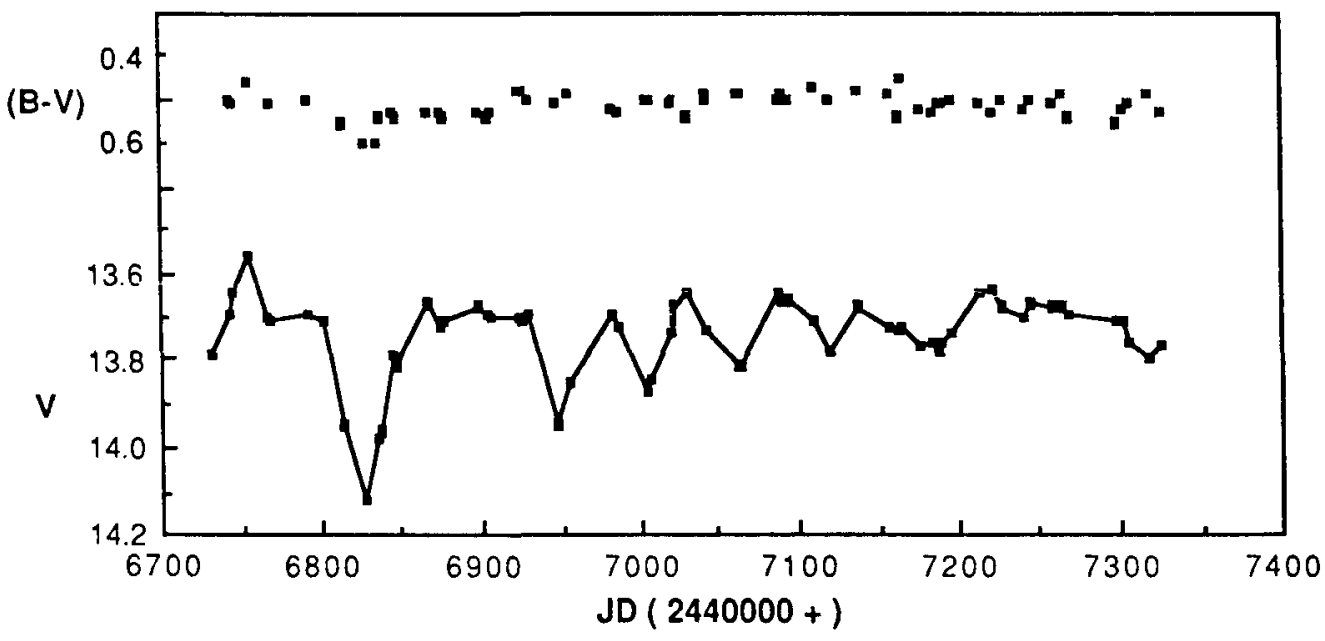

Figure 1. (B-V) and V curves for HV12842. 